\title{
Solar activity in the last millennium: inductive reconstructions from proxy data
}

\author{
E.V. Miletsky ${ }^{1}$, V.G. Ivanov ${ }^{1}$, Yu. A. Nagovitsyn ${ }^{1}$, H. Jungner ${ }^{2}$ \\ ${ }^{1}$ Central Astronomical Observatory at Pulkovo, Saint-Petersburg, Russia, e-mail: \\ solar1@gao.spb.ru \\ ${ }^{2}$ University of Helsinki, Finland, e-mail: hogne.jungner@helsinki.fi
}

\begin{abstract}
The average sunspot activity level for the last millennium is reconstructed. There is a good agreement between the reconstructions, independently built by different proxies $\dagger$.
\end{abstract}

The objective of this paper is reconstruction of solar activity in the past with time scale longer than 30 years. For this purpose we combine all available proxies: cosmogenic isotopes concentrations $\left({ }^{14} \mathrm{C}\right.$ and $\left.{ }^{10} \mathrm{Be}\right)$ and data of historical chronicles, accounting all independent information from them and thereby increasing reliability of the reconstructions.

Below we use series of cosomogenic isotope ${ }^{10} \mathrm{Be}(\mathrm{BeSP})$ concentration from Antarctic ice probes (Bard et al. 1997, Raisbeck et al. 1990) for period $850-1982$. Variations of ${ }^{14} \mathrm{C}$ concentration are estimated with use of "residuals" $\delta^{14} \mathrm{C}$, that reflect abundances of the isotope above a certain natural level in the corresponding epoch. We use the series of the residuals (C14) that contain decade data from 9665 BC to 1945 AD (Stuiver et al. 1998). For reconstruction we select from the series data in the interval 850-1900, to exclude antropogenic factors in the 20th century. Since aurorae are connected with processes of geomagnetic and solar activity, one can use as proxies of solar activity historical chronicles that contain information about observed middle- and low-latitude aurorae. We use these data, that recently were compiled and extended by Krivsky (1988) and Silverman (2002), for the period 850-1900 ( $\mathrm{Au})$. One more proxy of the solar activity is the historical records of naked-eye sunspot observations that are compiled by Wittmann \& Xu (1987). For reconstruction we limit ourselves with the time interval of the corresponding series (WX) since 850 to 1900.

All the series were interpolated to one-year time step. The long-term trend component of $\mathrm{C} 14$ series that corresponds to time scale more than 1500 years were obtained by polynomial fitting and subtracted from the series. The aurorae series Au was normalized with use of dividing by 200-year running average in order to remove the trend caused by loss of information as moving to the past. To reduce noise from short-term components, the obtained series were processed by 13 -year running averaging with harmonic weights. The models were built with account for possible time lag in some proxy series relative to the solar activity. It proved to be operative to transform all series to five-year step, calculating corresponding 5 -point means. The same operations were applied to two known "reference" series that reflects solar activity in the instrumental epoch, namely Wolf sunspot numbers (WSN) (1700-1995) and group sunspot number by Hoyt and Schatten (1998) (GSN) (1610-1995). We made investigation that enable us to choice the Wolf sunspot numbers (WSN) as a main reference index of the solar activity, regardless of the fact that the GSN series is longer.

$\dagger$ The full version of this paper can be found at http://www.gao.spb.ru/personal/ivanov/ papers/mil-iva-iaus2004.pdf 


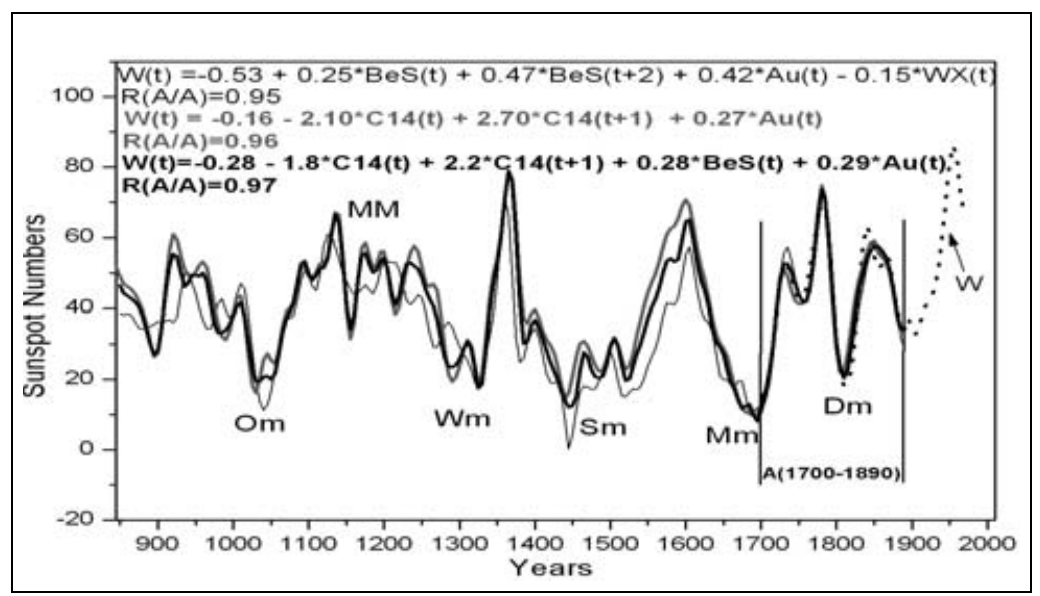

Figure 1. Set of "combined" models with different combinations of proxies

For construction of models we use method of "Inductive Self-organizing Modeling" (ISM) (Farlow 1984, Madala \& Ivakhnenko 1994). The method allows obtaining, on the base of empirical data, models of optimal complexity, which in a best way correspond to a certain selected criterion.

A good agreement exists between reconstructions that are made with use of "combined" models including different combinations of proxies (see Figure). All these reconstructions present well the known epochs of extreme solar activity. The most accurate one (correlation coefficient $R=0.97$ ) on interval $1700-1890$ is a model that includes three proxies of the four (BeS, $\mathrm{C} 14$ and $\mathrm{Au}$ ). In this model the Maunder minimum turns to be deepest, and the global maxima of activity are only slightly lower then the maximum in the 20th century. The maxima in all of these reconstructions prove to be essentially higher than in reconstruction of Usoskin et al. (2004).

The obtained results demonstrate effectiveness of the approach to reconstruction of the solar activity in the past that is based upon using of different, supplementing each other, solar activity proxies. The choice of optimal and accurate models with accounting of time lags are supplied by modern method of inductive modeling.

The work was supported by grants INTAS 2000-0752, INTAS 2001-0550, RFBR 0402-17560 and RFBR 03-02-17505, Federal Program "Astronomy-1105", Program of Presidium of Russian Academy of Sciences "Non-stationary phenomena in astronomy".

\section{References}

Bard, E., Raisbek, G.M., Yiou, F. \& Jouzel, J. 1997 Earth and Planet. Sci. Lett. 150, 453.

Farlow, S. J. (ed.) 1984 Self-organizing Method in Modeling: GMDH Type Algorithms. Statistics: Textbooks and Monographs $\mathbf{5 4 .}$

Hoyt D.V., \& Schatten, K. 1998 Solar Phys. 179189.

Krivsky, L. \& Pejml, K. 1988 Publ. Astr. Ist.Czech. 75.

Madala,H.R. \& Ivakhnenko,A.G. 1994 In Inductive Learning Algorithms for Complex Systems Modeling. CRC Press Inc., Boca Raton. 115.

Raisbeck, G.M., Yiou, F.Y., Jouzel, J., \& Petit, J.R. 1990 Phil. Trans. R. Soc. Lond. A330, 463.

Silverman, S.M., 2002 J. Atmos. Solar-Terr. Phys. 63, 523.

Stuiver, M., P. J. Reimer \& T. F. Braziunas. 1998 Radiocarbon 40, 1127.

Usoskin, I.G. et al. 2004 Reply, Phys. Rev. Lett. 92(19), 199002.

Wittmann, A.D \& Xu, Z.T. 1987 Astron. Astrophys. Suppl.Ser. 7083. 